\title{
Relations as Abstract Datatypes: An Institution to Specify Relations between Algebras*
}

\author{
Hubert Baumeister \\ Max-Planck-Institut für Informatik \\ Im Stadtwald, 66123 Saarbrücken, Germany \\ hubertompi-sb.mpg . de
}

\begin{abstract}
One way to view the execution state of an imperative program is as a many sorted algebra. Program variables are (constant) functions and their types are sorts. The execution of a program defines a relation between the state of the program (algebra) before and after the execution of the program. In this paper we shall define an institution for the specification of relations between structures of some base institution (eg. the institution of equational logic or first order predicate logic). Sets of structures over a common signature, abstract datatypes, in this institution denote relations between structures of the base institution. This makes it possible to apply a rich repertoire of existing techniques for specifying abstract datatypes to the specification of relations. This paper tries to narrow the gap between algebraic specification languages like Clear, ASL or Act-One and model theoretic based specification languages like Z, VDM-SL or the Larch Interface language.
\end{abstract}

\section{Introduction}

States consisting of variables $x_{1} \ldots x_{n}$ of sort $s_{1} \ldots s_{m}$ can be seen as algebras over a many sorted signature $\Sigma=\langle S, O p\rangle$ having sorts $S=\left\{s_{\imath} \mid i=1 \ldots m\right\}$ and operations $O p=\left\{x_{\imath}: s_{i} \mid i=1 \ldots n, s_{\imath} \in S\right\}$, possibly satisfying some state invariants.

As an example consider the specification of a birthday book, taken from the $\mathrm{Z}$ reference manual [17]. The goal is to specify a system to record people's birthdays. The state of a birthday book consists of known, a set of names of peoples whose birthdays are recorded, and birthday, a set of associations of names to dates. The state invariant is that known is exactly the set of names occuring as a first component in the elements of birthday. Using a notation adapted from $Z$, the state of a birthday book is described by the specification Birthdaybook

"The research described in this paper was supported by the German Ministry for Research and Technology (Bundesministerium für Forschung und Technologie) under grant ITS 9103 and by the ESPRIT Basic Research Working Group 6112 COMPASS (A Comprehensive Algebraic Approach to System Specification and Development). 
Birthdaybook

Env

known : SETOFNAME

birthday : SETOFBBENTRY

$\forall n: N A M E \quad n \in$ known iff

$\exists$ entry : BBENTRY entry $\in$ birthday and name $($ entry $)=n$

Birthdaybook is based on the specification Env

$\left[\begin{array}{l}\text { Env } \\ \text { BBEntry } \\ \text { Set }(\text { Name })[\text { SETOFNAME } \leftarrow \text { SET }] \\ \text { Set }(\text { BBEntry })[\text { SETOFBBENTRY } \leftarrow \text { SET }]\end{array}\right.$

and BBEntry

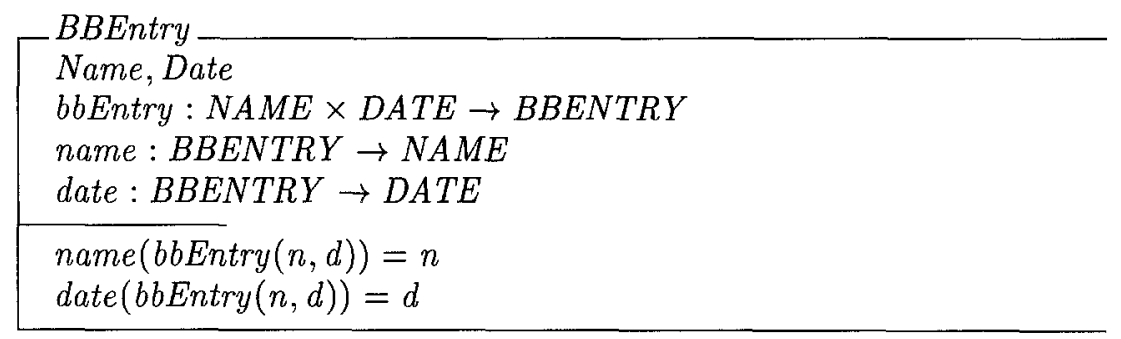

and where the specifications Name and Date merely define the sorts $N A M E$ and DATE. Set (Name)[SETOFNAME $\leftarrow S E T]$ is the notation for the application of the parameterized specification Set to the specification Name and then renaming the sort SET in the result to SETOFNAME. ${ }^{2}$ Algebras satisfying the Birthdaybook specification are possible states of a birthday book.

Operations modifying the state of a system are interpreted as functions on algebras or, more generally, as relations between algebras. For example, the operation AddBirthday (name: NAME; date:DATE) adds a new entry to the birthday book if the birthday for name is not already known. More precisely, $\operatorname{AddBirthday}(n, d)$ defines, for a name $n$ and a date $d$, a relation between Birthdaybook algebras $A$ and $B$ such that known ${ }^{B}=k_{n o w n}{ }^{A} \cup\{n\}$, birthday ${ }^{B}=$ birthday $^{A} \cup\left\{b b\right.$ Entry $\left.{ }^{A}(n, d)\right\}$ and such that $A$ and $B$, when restricted to their environment, the specification $E n v$, coincide.

More formally we may define specifications

AddBirthdayIn

Birthdaybook

name? : NAME

date? : DATE

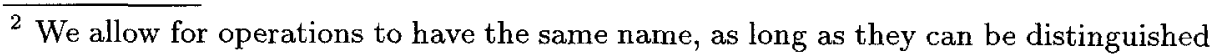
by their types. For instance the insert operations from Set (Name)[SETOFNAME $S E T]$ and Set (BBEntry) [SETOFBBENTRY $\leftarrow S E T]$ are considered different. 
and

AddBirthdayOut

Birthdaybook

and consider AddBirthday as a relation between $A d d B i r t h d a y I n$ algebras $A$ and $A d d B$ irthdayOut algebras $B$ satisfying $\left.A\right|_{E n v}=\left.B\right|_{E n v}$ and the equations

$$
\begin{aligned}
& \text { known }^{B}=\text { known }^{A} \cup\left\{\text { name }{ }^{A}\right\} \text { and } \\
& \text { birthday }{ }^{B}=\text { birthday }^{A} \cup\left\{b b \text { Entry }{ }^{A}\left(\text { name }{ }^{A}{ }^{A} \text {, date } ?^{A}\right)\right\} .
\end{aligned}
$$

The additional constants name? and date? in AddBirthdayIn model the input parameters of the operation AddBirthday. In case AddBirthday is supposed to deliver a result $x$ ! of sort $s$, a constant $x !: s$ is added to AddBirthdayOut. Next we observe that $A$ and $B$ can be extended to an algebra $C$, a model of

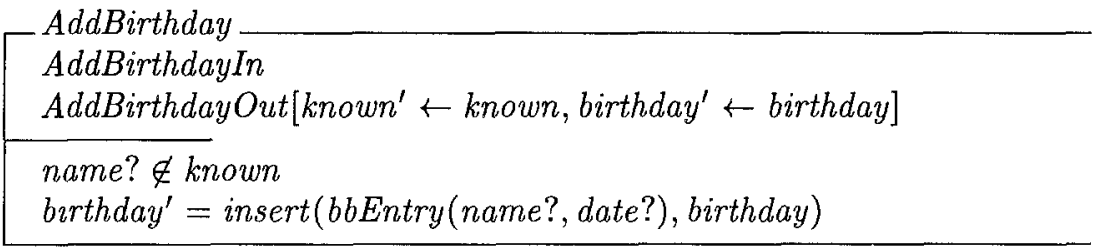

such that

$$
\left.C\right|_{\text {AddBirthdayIn }}=A \text { and }\left.C\right|_{\text {AddBirthdayOut }}=B \text {. }
$$

Note, that there is no need to include an axiom of the form

$$
\text { known' }=\operatorname{insert}(\text { name?, known) }
$$

explicitly in AddBirthday because this is a consequence of the axiom

$$
\text { birthday' }=\text { insert (bbEntry (name?, date?), birthday) }
$$

together with the axioms of Birthdaybook.

On the other hand, the specification AddBirthday defines a relation $R$ between states of birthday book (models of Birthdaybook) by

$$
A R B \quad \text { iff } \quad \exists C \quad C \models \text { AddBirthday, }\left.C\right|_{\iota}=A \text { and }\left.C\right|_{\sigma}=B \text {, }
$$

where $\iota$ is the inclusion of Birthdaybook into AddBirthday and $\sigma$ is a morphism from Birthdaybook to AddBirthday mapping known to known' and birthday to birthday'. Therefore, relations between algebras can be regarded as sets of algebras specified using standard abstract datatypes techniques.

In the rest of this paper we shall define an institution $\mathcal{R}_{\mathbf{D}}$ over some base institution $\mathcal{I}$ such that sets of formulas in this institution denote relations between structures from $\mathcal{I}$, which formally are abstract datatypes in the institution $\mathcal{R}_{\mathbf{D}}$. Abstract datatypes are pairs $\langle\Sigma, M\rangle$, where $\Sigma$ is a signature and $M$ a set of $\Sigma$-structures. We shall show that this institution inherits some nice properties from its base institution, like for example a cocomplete category of signatures and amalgamation, which implies extension. 


\section{Preliminaries}

\subsection{Institutions}

The notion of an institution was introduced by Goguen and Burstall to define the semantics of the specification language Clear [3] independent of an underlying logic. Here we will only recall the definition of institutions, for more details see the paper by Goguen and Burstall [10]. An institution $\mathcal{I}=\langle S I G N, \operatorname{Mod}, \operatorname{Sen}, \vDash\rangle$ consists of

- a category of signatures $S I G N$,

- a functor Mod:SIGN $\rightarrow C A T^{o p}$ assigning to each signature $\Sigma$ the category of $\Sigma$-structures and to each signature morphism $\sigma: \Sigma \rightarrow \Sigma^{\prime}$ a forgetful functor $\operatorname{Mod}(\sigma): \operatorname{Mod}\left(\Sigma^{\prime}\right) \rightarrow \operatorname{Mod}(\Sigma),{ }^{3}$

- a functor Sen:SIGN $\rightarrow S E T$, assigning to each signature $\Sigma$ the set of $\Sigma$ formulas and to each signature morphism $\sigma: \Sigma \rightarrow \Sigma^{\prime}$ a translation $\operatorname{Sen}(\sigma)$ of $\Sigma$-formulas to $\Sigma^{\prime}$-formulas, ${ }^{4}$ and

- a family of satisfaction relations $\left(\models_{\Sigma} \subseteq \operatorname{Mod}(\Sigma) \times \operatorname{Sen}(\Sigma)\right)_{\Sigma \in S I G N}$ indicating whether a $\Sigma$-formula $f$ is valid in a $\Sigma$-structure $m\left(m \models_{\Sigma} f\right.$ or for short $m \vDash f$ )

where the satisfaction condition holds: for all signature morphisms $\sigma: \Sigma \rightarrow \Sigma^{\prime}$, formulas $f \in \operatorname{Sen}(\Sigma)$ and structures $m^{\prime} \in \operatorname{Mod}\left(\Sigma^{\prime}\right)$

$$
\left.m^{\prime}\right|_{\sigma} \models f \quad \text { iff } \quad \mathrm{m}^{\prime} \vDash \sigma(\mathbf{f})
$$

Each institution has associated two categories $P R E S_{\mathcal{I}}$ and $A D T_{\mathcal{I}}$. The objects of $P R E S_{\mathcal{I}}$ are pairs $\langle\Sigma, F\rangle$ where $\Sigma$ is a signature from $S I G N$ and $F$ a set of $\Sigma$-formulas. Morphisms $\sigma:\langle\Sigma, F\rangle \rightarrow\left\langle\Sigma^{\prime}, F^{\prime}\right\rangle$ in $P R E S_{\mathcal{I}}$ are signature morphisms such that $F^{\prime} \models \sigma(F)$. In $A D T_{\mathcal{I}}$ objects are pairs $\langle\Sigma, M\rangle$ where $M$ is a set of $\Sigma$ structures and morphisms $\sigma:\langle\Sigma, M\rangle \rightarrow\left\langle\Sigma^{\prime}, M^{\prime}\right\rangle$ are signature morphisms such that $\left.M^{\prime}\right|_{\sigma} \subseteq M$. There are also forgetful functors $S i g^{p}: P R E S_{\mathcal{I}} \rightarrow S I G N$ and $S i g^{a}: A D T_{\mathcal{I}} \rightarrow S I G N$ defined by

$$
\operatorname{Sig}^{p}(\langle\Sigma, F\rangle)=\operatorname{Sig}^{a}(\langle\Sigma, M\rangle)=\Sigma \text { and } \operatorname{Sig}^{p}(\sigma)=\operatorname{Sig}^{a}(\sigma)=\sigma
$$

It is known (eg. $[15,2]$ ) that the functors $\operatorname{Sig}^{p}$ and Sig $^{a}$ create (finite) (co)limits and that as a consequence $P R E S_{\mathcal{I}}$ and $A D T_{\mathcal{I}}$ are (finitely) (co)complete if the category of signatures $S I G N$ is.

Objects in $P R E S_{\mathcal{I}}$ can be thought of as specifying or presenting objects in $A D T_{\mathcal{I}}$, which are called abstract datatypes. Thus, there is a semantics function sem: $P R E S_{\mathcal{I}} \rightarrow A D T_{\mathcal{I}}$ associating to a presentation $\langle\Sigma, F\rangle$ an abstract datatype $\left\langle\Sigma^{\prime}, M^{\prime}\right\rangle$. Two commonly used semantic functions are loose and initial, where

$$
\operatorname{loose}(\langle\Sigma, F\rangle)=\langle\Sigma,\{m \in \operatorname{Mod}(\Sigma) \mid m \models F\}\rangle
$$

and initial $(\langle\Sigma, F\rangle)=\langle\Sigma, M\rangle$ with $M$ the set of all objects that are initial in the full subcategory of $\operatorname{Mod}(\Sigma)$ whose objects satisfy $F$.

\footnotetext{
${ }^{3}$ The application of $\operatorname{Mod}(\sigma)$ to a $\Sigma^{\prime}$-structure $m^{\prime}$ will be written as $\left.m^{\prime}\right|_{\sigma}$. If $\sigma$ is understood from the the context we may write $\left.m^{\prime}\right|_{\Sigma}$ instead.

${ }^{4}$ The application of $\operatorname{Sen}(\sigma)$ to a formula $f$ will be abbreviated by $\sigma(f)$.
} 


\section{$2.2 \quad$ Limits and Colimits}

This section defines the notations used in this paper for limits and colimits in categories. For a definition of limits and colimits refer, for example, to the book of MacLane [14].

We use the notation $\prod_{\mathbf{I}} F$ to denote the limit object of the functor $F: \mathbf{I} \rightarrow \mathbf{C}$, if it exists, and $\pi_{i}^{F}$, or $\pi_{i}$ if there is no ambiguity, for the projections $\pi_{i}^{F}: \prod_{\mathbf{I}} F \rightarrow$ $F(i)$. If $G: \mathbf{I} \rightarrow \mathbf{C}$ is another functor with a limit and $\tau: F \Rightarrow G$ is a natural transformation, then $\prod_{\mathbf{I}} \tau$ denotes the unique morphism from the limit object $\prod_{\mathbf{I}} F$ to the limit object $\prod_{\mathbf{I}} G$ such that for all $i \in \mathbf{I}$ the following diagram commutes

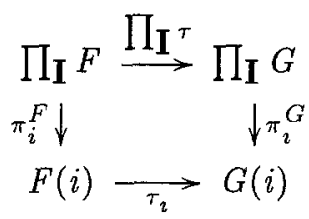

The colimit object of a functor $F: \mathbf{I} \rightarrow \mathbf{C}$ is denoted by $\sum_{\mathbf{I}} F$ and $\iota_{\imath}^{F}$, or $\iota_{\imath}$, denotes the inclusions $\iota_{\imath}^{F}: F(i) \rightarrow \sum_{\mathbf{I}} F$. If $G: \mathbf{I} \rightarrow \mathbf{C}$ is another functor with a colimit and $\tau: F \Rightarrow G$ is a natural transformation, then $\sum_{\mathbf{I}} \tau$ denotes the unique morphism from the colimit object $\sum_{\mathbf{I}} F$ to the colimit object $\sum_{\mathbf{I}} G$ such that for all $i \in \mathbf{I}$ the following diagram commutes

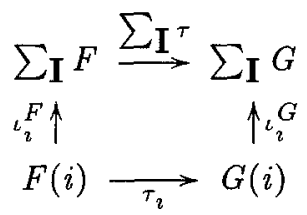

\section{Binary Relations}

Before presenting the general construction in section 4 we shall illustrate the construction using binary relations. Let us assume we are given an institution $\mathcal{I}=\langle$ SIGN, Mod, Sen, $\models\rangle$ and let $\Theta=\left\langle\Sigma_{1}, \Sigma_{2}\right\rangle$ be a pair of signatures from SIGN. A (binary) relation $R$ of type $\Theta$ is a subset $M$ of the cartesian product of $\operatorname{Mod}\left(\Sigma_{1}\right)$ and $\operatorname{Mod}\left(\Sigma_{2}\right)$. A morphism $\alpha: R \rightarrow S$ from a relation $R$ of type $\Theta^{R}$ to a relation $S$ of type $\Theta^{S}$ is a pair of signature morphisms $\alpha_{1}: \Sigma_{1}^{R} \rightarrow \Sigma_{1}^{S}$ and $\alpha_{2}: \Sigma_{2}^{R} \rightarrow \Sigma_{2}^{S}$ such that whenever $m_{1} S m_{2}$ then $\left.\left.m_{1}\right|_{\alpha_{1}} R m_{2}\right|_{\alpha_{2}}$. The category $R E L$ of binary relations has relations as objects and morphisms between relations as arrows.

To specify objects from $R E L$ we need to define a notion of formulas together with a satisfaction relation between formulas and elements of the cartesian product of $\operatorname{Mod}\left(\Sigma_{1}\right)$ and $\operatorname{Mod}\left(\Sigma_{2}\right)$. A possible definition of such formulas is as elements of the disjoint union of $\operatorname{Sen}\left(\Sigma_{1}\right)$ and $\operatorname{Sen}\left(\Sigma_{2}\right)$. Then for a pair $\left\langle m_{1}, m_{2}\right\rangle$ of $\operatorname{Mod}\left(\Sigma_{1}\right) \times \operatorname{Mod}\left(\Sigma_{2}\right)$ and a formula $f$ of $\operatorname{Sen}\left(\Sigma_{1}\right)+\operatorname{Sen}\left(\Sigma_{2}\right)$ we define

$$
\left\langle m_{1}, m_{2}\right\rangle \models^{R E L} f \quad \text { iff } \quad\left\{\begin{array}{l}
m_{1} \models f \text { if } f \in \operatorname{Sen}\left(\Sigma_{1}\right) \\
m_{2} \vDash f \text { if } f \in \operatorname{Sen}\left(\Sigma_{2}\right)
\end{array}\right.
$$


However, with this definition it is not possible to write a formula similar to

$$
\text { birthday' }=\text { insert (bbEntry (name?, date?), birthday })
$$

from the AddBirthday example because this formula is neither a formula over the signature of AddBirthdayIn nor a formula over the signature of AddBirthdayOut, instead, it is a formula over the disjoint union of the signatures of $A d d B i r t h d a y I_{n}$. and AddBirthdayOut.

Therefore, we are interested in formulas $f$ from $\operatorname{Sen}\left(\Sigma_{1}+\Sigma_{2}\right)$. Now we have the problem to define the satisfaction relation between pairs $\left\langle m_{1}, m_{2}\right\rangle$ and formulas $f$. In the institution of first order logic, and many other interesting institutions, there is a one to one correspondence between pairs of algebras from $\operatorname{Mod}\left(\Sigma_{1}\right) \times \operatorname{Mod}\left(\Sigma_{2}\right)$ and algebras from $\operatorname{Mod}\left(\Sigma_{1}+\Sigma_{2}\right)$. A $\Sigma_{1}+\Sigma_{2}$-algebra $m$ defines a pair of $\Sigma_{1}$ - and $\Sigma_{2}$-algebras by $\left\langle\left. m\right|_{\Sigma_{1}},\left.m\right|_{\Sigma_{2}}\right\rangle$ and each pair $\left\langle m_{1}, m_{2}\right\rangle$ defines an algebra $m_{1}+m_{2}$ such that the interpretation of a symbol $s$ (a sort, function or predicate symbol) from $\Sigma_{1}+\Sigma_{2}$ in $m_{1}+m_{2}$ is the interpretation of $s$ in $m_{1}$ if $s$ originates from $\Sigma_{1}$ or the interpretation of $s$ in $m_{2}$ otherwise. This makes it possible to define

$$
\left\langle m_{1}, m_{2}\right\rangle \models{ }^{R E L} f \quad \text { iff } \quad m_{1}+m_{2} \vDash f .
$$

Given a set of formulas $F$ from $\operatorname{Sen}\left(\Sigma_{1}+\Sigma_{2}\right)$ a pair $S p=\langle\Theta, F\rangle$ defines a relation by

$$
m_{1} S p m_{2} \quad \text { iff } \quad \forall f \in F:\left\langle m_{1}, m_{2}\right\rangle \models^{R E L} f .
$$

The category of specifications of binary relations, RELSPEC, has as objects pairs $S p=\langle\Theta, F\rangle$ and as arrows $\alpha: S p_{R} \rightarrow S p_{S}$ signature morphisms $\alpha: \Sigma_{1}^{R}+\Sigma_{2}^{R} \rightarrow$ $\Sigma_{1}^{S}+\Sigma_{2}^{S}$ such that $F^{S} \models^{R E L} \alpha\left(F^{R}\right)$.

There is a functor Rel from RELSPEC to REL mapping specifications of binary relations to binary relations defined by

$$
\begin{gathered}
\operatorname{Rel}(\langle\Theta, F\rangle)=\left\langle\Theta,\left\{\left\langle m_{1}, m_{2}\right\rangle \mid\left\langle m_{1}, m_{2}\right\rangle \models^{R E L} F\right\}\right\rangle \text { and } \\
\operatorname{Rel}\left(\alpha: S p_{R} \rightarrow S p_{S}\right)=\alpha: \operatorname{Rel}\left(S p_{R}\right) \rightarrow \operatorname{Rel}\left(S p_{S}\right) .
\end{gathered}
$$

The domain of a binary relation $R=\langle\Theta, M\rangle$ in $R E L$ is given by

$$
\operatorname{dom}(R)=\left\{m_{1} \mid\left\langle m_{1}, m_{2}\right\rangle \in M\right\}
$$

and its codomain by

$$
\operatorname{cod}(R)=\left\{m_{2} \mid\left\langle m_{1}, m_{2}\right\rangle \in M\right\} .
$$




\subsection{Binary Relations as Abstract Datatypes}

In this section we shall define an institution $\mathcal{R}_{\mathbf{2}}$ such that the category of abstract datatypes ${ }^{5} A D T_{2}$ (cf. section 2.1) in this institution is the category of binary relations $R E L$ and the category of presentations $P R E S_{2}$ is the category of specifications of binary relations $R E L S P E C$. The construction of $\mathcal{R}_{2}$ is parameterized by an institution $\mathcal{I}$. To define the satisfaction relation of $\mathcal{R}_{\mathbf{2}}$ we require that SIGN has coproducts and that Mod preserves them. Now the institution

$$
\mathcal{R}_{2}=\left\langle R S I G N 2, \operatorname{RMod} 2, R \operatorname{Sen} 2, \models^{\mathcal{R}_{2}}\right\rangle
$$

is given by:

$$
\begin{gathered}
r S I G N 2=S I G N^{\mathbf{2}}, \\
R M o d 2\left(\Theta: \mathbf{2} \rightarrow \operatorname{SIGN)}=\prod_{\mathbf{2}} \Theta ; \operatorname{Mod} \quad R \operatorname{RMod} 2\left(\alpha: \Theta^{R} \Rightarrow \Theta^{S}\right)=\prod_{\mathbf{2}} \alpha ; \operatorname{Mod}\right. \\
R \operatorname{Sen} 2(\Theta)=\operatorname{Sen}\left(\sum_{\mathbf{2}} \Theta\right) \quad \operatorname{RSen} 2(\alpha)=\operatorname{Sen}\left(\sum_{\mathbf{2}} \alpha\right) \\
m \models^{\mathcal{R}_{2} f} \quad \text { iff } \quad m \vDash f
\end{gathered}
$$

The category 2 is the discrete category having only 1 and 2 as objects and the identities as arrows.

Objects in $A D T_{\mathbf{2}}$ are pairs $R=\langle\Theta, M\rangle$, where $\Theta$ is an element of $R S I G N 2$ and $M$ is a subset of $R M o d 2(\Theta)$. $\Theta$ is a functor from 2 to $S I G N$ and thus, a pair of signatures $\langle\Theta(1), \Theta(2)\rangle$ from $\operatorname{SIGN} \times \operatorname{SIGN}$. Since $R \operatorname{Mod} 2(\Theta)$ is $\operatorname{Mod}(\Theta(1)) \times$ $\operatorname{Mod}(\Theta(2))$, elements of $M \subseteq R M o d 2(\Theta)$ are pairs $\left\langle m_{1}, m_{2}\right\rangle$.

An arrow $\alpha: \Theta^{R} \Rightarrow \Theta^{S}$ in RSIGN2 is a natural transformation between functors from 2 to $S I G N$ and thus, just a pair of signature morphisms $\alpha_{2}$ from $\Theta^{R}(i)$ to $\Theta^{S}(i)(i=1,2)$. The application of $R M$ Mod 2 to an arrow $\alpha$ of $R S I G N 2$ yields the pair of functors $\left\langle\operatorname{Mod}\left(\alpha_{1}\right), \operatorname{Mod}\left(\alpha_{2}\right)\right\rangle$.

Thus an arrow $\alpha:\left\langle\Theta^{R}, M^{R}\right\rangle \rightarrow\left\langle\Theta^{S}, M^{S}\right\rangle$ in $A D T_{\mathbf{2}}$ is a morphism $\alpha$ from $\Theta^{R}$ to $\Theta^{S}$ in $R S I G N 2$ such that $\left\langle\left. m_{1}\right|_{\alpha_{1}},\left.m_{2}\right|_{\alpha_{2}}\right\rangle \in M^{R}$ for all $\left\langle m_{1}, m_{2}\right\rangle \in M^{S}$ or, whenever $m_{1} S m_{2}$ then $\left.\left.m_{1}\right|_{\alpha_{1}} R m_{2}\right|_{\alpha_{2}}$.

Since $S I G N$ has coproducts, the object $\sum_{2} \Theta=\Theta(1)+\Theta(2)$ exists for any object $\Theta$ in RSIGN2. Moreover, since Mod preserves coproducts

$$
\operatorname{Mod}(\Theta(1)+\Theta(2))=\operatorname{Mod}(\Theta(1)) \times \operatorname{Mod}(\Theta(2))=\operatorname{RMod} 2(\Theta) .
$$

Therefore each $m$ in $R \operatorname{Mod} 2(\Theta)$ is also in $\operatorname{Mod}(\Theta(1)+\Theta(2))$ and each formula $f$ in $R \operatorname{Sen} 2(\Theta)$ is an element of $\operatorname{Sen}(\Theta(1)+\Theta(2))$. Thus the satisfaction relation $m \models^{\mathcal{R}_{2}} f$ iff $m \models f$ is well defined.

\footnotetext{
${ }^{5}$ To avoid subscripts of subscripts we write $A D T_{\mathbf{2}}$ instead of $A D T_{\mathcal{R}_{\mathbf{2}}}$.
} 


\section{An Institution to Specify Relations Between Algebras}

The institution $\mathcal{R}_{\mathbf{2}}$ is based on the discrete category 2 . However, the construction of $\mathcal{R}_{2}$ did not depend on the specific properties of 2 . In fact, the same construction can be used to define an institution

$$
\mathcal{R}_{\mathbf{D}}=\left\langle R S I G N D, \operatorname{RMod} D, R \operatorname{Sen} D, \models^{\mathcal{R}_{D}}\right\rangle
$$

based on an arbitrary category $\mathbf{D}$, provided that $S I G N$ is cocomplete with respect to diagrams of shape $\mathbf{D}$ and that Mod preserves the colimit of diagrams of shape D:

$$
\begin{aligned}
& R S I G N D=S I G N^{\mathbf{D}} \\
& R \operatorname{Mod} D(\Theta: \mathbf{D} \rightarrow S I G N)=\prod_{\mathbf{D}} \Theta ; \operatorname{Mod} \quad R \operatorname{Mod} D\left(\alpha: \Theta^{R} \Rightarrow \Theta^{S}\right)=\prod_{\mathbf{D}} \alpha ; \operatorname{Mod} \\
& R \operatorname{Sen} D(\Theta)=\operatorname{Sen}\left(\sum_{\mathbf{D}} \Theta\right) \quad R \operatorname{Sen} D(\alpha)=\operatorname{Sen}\left(\sum_{\mathbf{D}} \alpha\right) \\
& m \models^{\mathcal{R}_{D} f} \quad \text { iff } \quad m \models f
\end{aligned}
$$

The following easy facts hold:

$-\mathcal{R}_{\mathbf{D}}$ is an institution,

- RSIGND is (finitely) cocomplete if $S I G N$ is and

- RModD preserves (finite) colimits if Mod preserves them.

Objects in $A D T_{\mathbf{D}}$ are typed relations of arity $\mathbf{D}$. Let, for example, $\mathbf{D}$ be the discrete category $\mathbf{K}_{n}$ generated by the set $\{1, \ldots, n\}$. An object $\Theta$ of $R S I G N K_{n}$ is an $n$-tuple $\Sigma_{1}, \ldots, \Sigma_{n}$ of signatures from $S I G N$ and objects in $R M O d K_{n}(\Theta)$ are $n$-tuples $m_{1}, \ldots, m_{n}$ such that $m_{i} \in \operatorname{Mod}\left(\Sigma_{2}\right)$. Thus an object $R=\langle\Theta, M\rangle$ in $A D T_{\mathbf{K}_{n}}$ is an $n$-ary relation $R \subseteq \operatorname{Mod}\left(\Sigma_{1}\right) \times \ldots \times \operatorname{Mod}\left(\Sigma_{n}\right)$.

Now let $\mathbf{D}$ have an arrow $g: d \rightarrow d^{\prime}$ other than the identity. Together with signatures $\Theta(d)=\Sigma_{d}$ and $\Theta\left(d^{\prime}\right)=\Sigma_{d^{\prime}}$ objects $\Theta$ in RSIGND also provide a signature morphism $\Theta(g)=\sigma: \Sigma_{d} \rightarrow \Sigma_{d^{\prime}}$. This imposes on objects $m$ from $R M o d D(\Theta)$ the restriction that

$$
\left.\pi_{d^{\prime}}(m)\right|_{\sigma}=\left.m_{d^{\prime}}\right|_{\sigma}=m_{d}=\pi_{d}(m)
$$

\section{Relations with Shared Parts}

We are interested in binary relations $R$, such that, if $m_{1} R m_{2}$ then $m_{1}$ and $m_{2}$ are persistent extensions of some algebra, like an algebra providing some base types such as bool and nat. This type of relation can be modeled by a three place relation $R$ given by a set $M \subseteq \operatorname{Mod}\left(\Sigma_{1}\right) \times \operatorname{Mod}\left(\Sigma_{0}\right) \times \operatorname{Mod}\left(\Sigma_{2}\right)$ with the requirement that for all $\left\langle m_{1}, m_{0}, m_{2}\right\rangle$ from $M$

$$
\left.m_{1}\right|_{\sigma_{1}}=m_{0}=\left.m_{2}\right|_{\sigma_{2}}
$$

where $\sigma_{\imath}: \Sigma_{0} \rightarrow \Sigma_{\imath}(i=1,2)$ define the parts that $\Sigma_{1}$ and $\Sigma_{2}$ have in common. 
The type of $R$ is given by a functor $\Theta$ from 3 to $S I G N$, where 3 is the category freely generated by the diagram

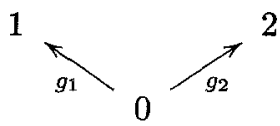

such that $\Theta(1)=\Sigma_{1}, \Theta(0)=\Sigma_{0}, \Theta(2)=\Sigma_{2}, \Theta\left(g_{1}\right)=\sigma_{1}$ and $\Theta\left(g_{2}\right)=\sigma_{2}$. The set $M$ is a subset of the objects of the pullback $\prod_{3} \Theta ; \operatorname{Mod}$ of the following diagram in $C A T$

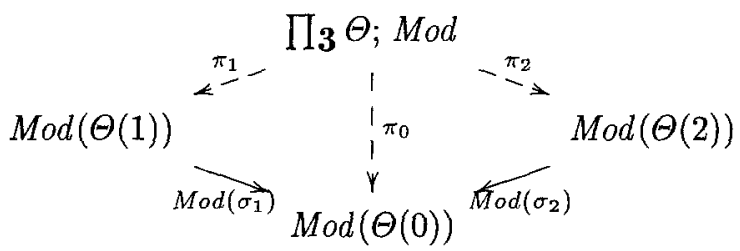

Since the above diagram commutes in $C A T$, for any object $m$ and morphism $h: m \rightarrow m^{\prime}$ in $\prod_{3} \Sigma ;$ Mod we have

$$
\begin{gathered}
\left.\pi_{1}(m)\right|_{\sigma_{1}}=\left.m_{1}\right|_{\sigma_{1}}=m_{0}=\left.m_{2}\right|_{\sigma_{2}}=\left.\pi_{2}(m)\right|_{\sigma_{2}} \text { and } \\
\left.\pi_{1}(h)\right|_{\sigma_{1}}=\left.h_{1}\right|_{\sigma_{1}}=h_{0}=\left.h_{2}\right|_{\sigma_{2}}=\left.\pi_{2}(h)\right|_{\sigma_{2}} .
\end{gathered}
$$

For objects $R=\langle\Theta, M\rangle$ in the category of abstract datatypes of the institution $\mathcal{R}_{3}$ we define

$$
m_{1} R m_{2} \quad \text { iff } \quad \exists m \in M: \pi_{1}(m)=m_{1} \text { and } \pi_{2}(m)=m_{2}
$$

which gives us that whenever $m_{1} R m_{2}$ then $\left.m_{1}\right|_{\Theta\left(g_{1}\right)}=\left.m_{2}\right|_{\Theta\left(g_{2}\right)}$.

Formulas in $R \operatorname{Sen} 3(\Theta)$ are formulas over the pushout $\sum_{\mathbf{3}} \Theta$ of the following diagram in $S I G N$

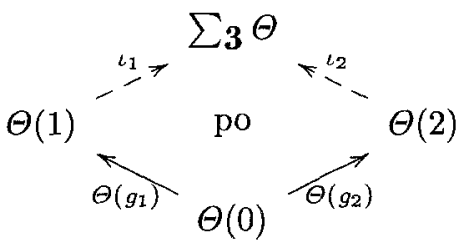

Here we have to require that $S I G N$ has all pushouts and that Mod preserves them, which is equivalent for the institution $\mathcal{I}$ to have amalgamation $[7,2,5]$.

As an example the AddBirthday specification is given as an object of $P R E S_{3}$ for first oder logic with equality as the base institution. The following functor $\Theta: \mathbf{3} \rightarrow S I G N$ is the signature of AddBirthday: 


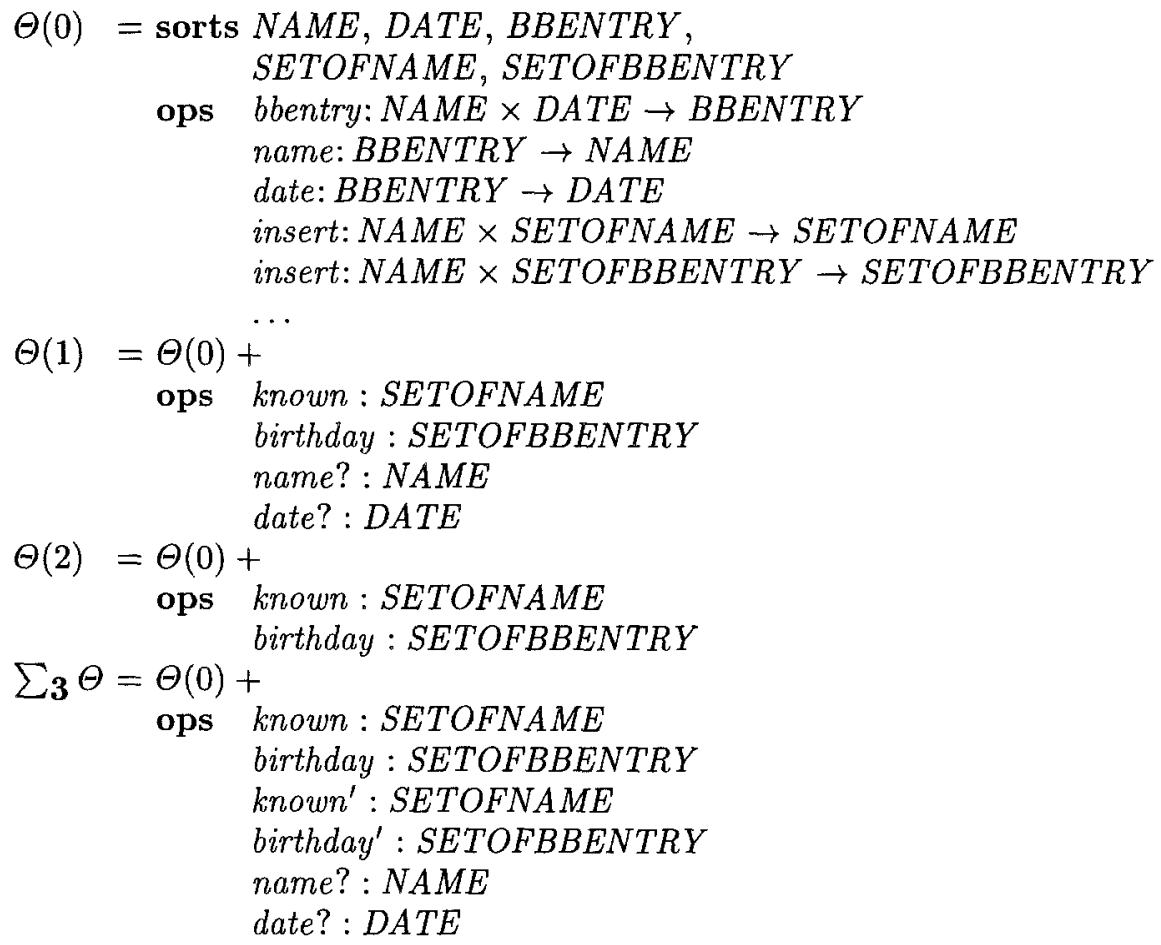

$\Theta\left(g_{1}\right), \Theta\left(g_{2}\right)$ and $\iota_{1}$ are the corresponding inclusion of signatures and $\iota_{2}$ maps birthday to birthday' and known to known' and is the identity on all other symbols. The axioms of AddBirthday are formulas in $R \operatorname{Sen} 3(\Theta)=\operatorname{Sen}\left(\sum_{\mathbf{3}} \Theta\right)$ :

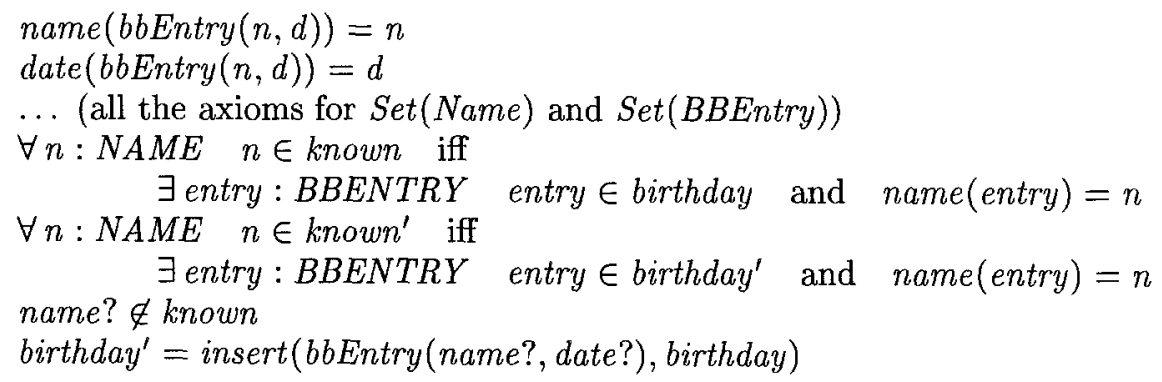

For an algebra $m$ of $R M o d 3(\Theta)$ satisfying AddBirthday it holds that, among others,

$$
\text { birthday }{ }^{m}=\operatorname{insert}^{m}\left(\text { bbEntry }{ }^{m}\left(\text { name } ?^{m}, \text { date }^{m}\right), \text { birthday }^{m}\right) .
$$

Since $m$ is a persistent extension of $m_{1}$ and $m_{2}$, which in turn are persistent extensions of $m_{0}$, this is equivalent to

$$
\text { birthday }^{m_{2}}=\operatorname{insert}^{m_{0}}\left(b_{b \text { Entry }}^{m_{0}}\left(\text { name } ?^{m_{1}}, \text { date } ?^{m_{1}}\right), \text { birthday }^{m_{1}}\right) .
$$

When writing specifications of relations it is convenient to use instead of functors $\Theta: \mathbf{D} \rightarrow S I G N$ functors $\Theta: \mathbf{D} \rightarrow P R E S_{\mathcal{I}}$ as the type of relations. We have 
done this in section 1, where we gave AddBirthdayIn, Env and AddBirthdayOut as specifications instead of just giving their signatures and including their axioms (eg. the axioms for the environment or the state invariant of BirthdayBook) in the set of formulas defining AddBirthday, as we have done in this section. This can be achieved by using the institution $\mathcal{P I}=\left\langle P R E S_{\mathcal{I}}, P M o d, S i g^{p} ;\right.$ Sen, $\left.\models\right\rangle$ built from an institution $\mathcal{I}$ as the base institution of $\mathcal{R}_{\mathrm{D}}$ instead of $\mathcal{I}$ itself. Here $\operatorname{PMod}(\langle\Theta, F\rangle)$ yields the full subcategory of $\operatorname{Mod}(\Theta)$ whose objects satisfy $F$.

\section{Specifications with Auxiliary Symbols}

In some cases we would like to use auxiliary sort or function symbols in the specification of relations. For example let $\Theta(1)$ and $\Theta(2)$ be enrichments of $\Theta(0)$ by a new sort $s$. To define how the interpretation of sort $s$ in $m_{1}$ is related to the interpretation of sort $s$ in $m_{2}$ an auxiliary function $f: \iota_{1}(s) \rightarrow \iota_{2}(s)$ may be needed. For example to require that $s^{m_{1}}$ is isomorphic to a subset of $s^{m_{2}}$ we provide the formula $\forall x, y: f(x)=f(y) \Rightarrow x=y$. However, this formula can not be written in any of the institutions $\mathcal{R}_{2}$ and $\mathcal{R}_{3}$ because formulas in these institutions are only allowed to use the symbols from $\Theta(1)$ and $\Theta(2)$.

A solution to this problem is to use $\mathcal{R}_{\mathbf{4}}$, where $\mathbf{4}$ is the category freely generated by

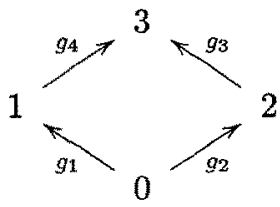

and the equation $g_{1} ; g_{4}=g_{2} ; g_{3}$. Now the function $f: \iota_{1}(s) \rightarrow \iota_{2}(s)$ can be defined in $\Theta(3)$. It is easy to prove that for a functor $\Theta: 4 \rightarrow$ SIGN from RSIGN4 the colimit $\sum_{4} \Theta$ is isomorphic to $\Theta(3)$. This holds for any category $\mathbf{D}$ with a terminal object $d_{T}$. Thus by providing $\Theta(3)$ one defines exactly the symbols one can use in the specification of relations. Most likely $\theta(3)$ will be an extension of the pushout of $\Theta\left(g_{1}\right)$ and $\Theta\left(g_{2}\right)$ by auxiliary sort and function symbols, but it is also possible that $\Theta(3), \Theta(1)$ and $\Theta(2)$ coincide.

Objects $S p=\langle\Theta, F\rangle$ in $P R E S_{4}$ define binary relations by

$$
m_{1} S p m_{2} \quad \text { iff } \quad \exists m \models^{\mathcal{R}_{4}} F: \pi_{1}(m)=m_{1} \text { and } \pi_{2}(m)=m_{2} .
$$

\subsection{Composition of Binary Relations}

Using $\mathcal{R}_{\mathbf{4}}$ has also the advantage that $P R E S_{4}$ is closed under the composition of binary relations. If $S p_{R}$ and $S p_{S}$ are viewed as binary relations and $S p_{R} ; S p_{S}$ is defined in the usual set theoretic way then there exists an object $S p_{T}$ of $P R E S_{4}$ defining the same binary relation as $S p_{R} ; S p_{S}$. This, depending on the expressiveness of the base institution $\mathcal{I}$, does not hold, in general, for the composition of relations in $P R E S_{2 / 3}$ (see below). 
Let $S p_{R}=\left\langle\Theta^{R}, F^{R}\right\rangle$ and $S p_{S}=\left\langle\Theta^{S}, F^{S}\right\rangle$ be specifications in $P R E S_{4}$. If $\Theta^{R}\left(g_{2}\right)=\Theta^{S}\left(g_{1}\right)$ then the composition $S p_{R} ; S p_{S}=\left\langle\Theta^{T}, F^{T}\right\rangle$ is defined. The type of $S p_{R} ; S p_{S}$ is given by the following diagram in $S I G N$

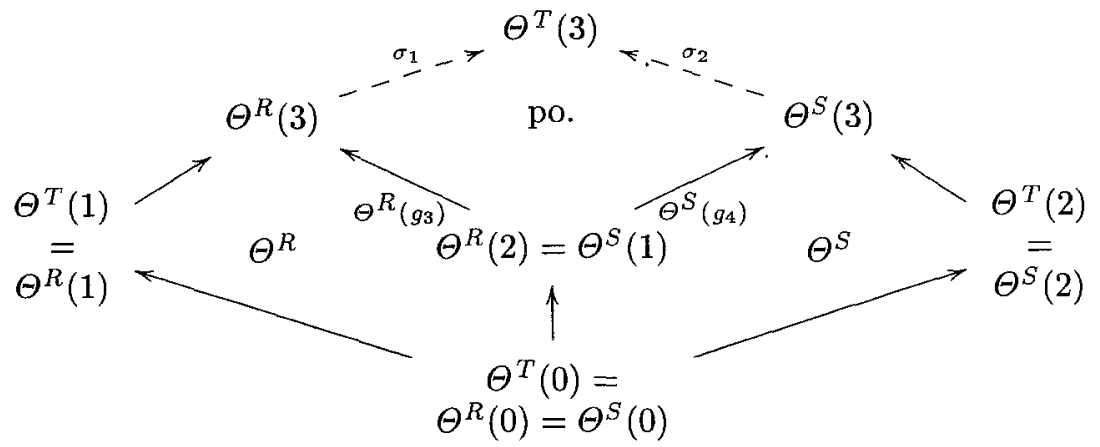

and $F^{T}=\sigma_{1}\left(F^{R}\right) \cup \sigma_{2}\left(F^{S}\right)$.

Theorem. $m_{1} S p_{R} ; S p_{S} m_{2} \quad$ iff $\quad \exists n: m_{1} S p_{R} n$ and $n S p_{S} m_{2}$

As an example consider the specifications $S p_{R}$ and $S p_{S}$ such that $\Theta^{R}\left(g_{2}\right)=$ $\Theta^{S}\left(g_{1}\right)$ and $\Theta^{R}(1)=\Theta^{S}(1)=\Theta^{S}(2)$ is an enrichment of $\Theta(0)$ by a constant $x: s$. Let $F^{R}$ contain the formula $x=f\left(x^{\prime}\right)$ and $F^{S}$ the formula $x^{\prime \prime}=g\left(x^{\prime}\right)$, where $f$ and $g$ are functions defined in $\Theta^{R}(0)$. For $S p_{R} ; S p_{S}=\left\langle\Theta^{T}, F^{T}\right\rangle$ as an object of $P R E S_{4}$ the type $\Theta^{T}$ contains the auxiliary symbol $x^{\prime}: s$ in $\Theta^{T}(3)$ together with $x: s$ from $\Theta^{T}(1)=\Theta^{R}(1)$ and $x^{\prime \prime}: s$ from $\Theta^{T}(2)=\Theta^{S}(2)$ and $F^{T}$ equals $\left\{x=f\left(x^{\prime}\right), x^{\prime \prime}=g\left(x^{\prime}\right)\right\}$.

However, to define $S p_{R} ; S p_{S}=\left\langle\Theta^{T}, F^{T}\right\rangle$ in $P R E S_{3}$ we do not have the possibility to use the auxiliary symbol $x^{\prime}: s$, therefore we have to find a set of formulas equivalent to the conjunction of $x=f\left(x^{\prime}\right)$ and $x^{\prime \prime}=g\left(x^{\prime}\right)$ only using the symbols $x, x^{\prime \prime}, f$ and $g$. Provided that the base institution $\mathcal{I}$ allows to use existential quantifiers we can use $\left\{\exists x^{\prime}: x=f\left(x^{\prime}\right) \wedge x^{\prime \prime}=g\left(x^{\prime}\right)\right\}$. In general, it may not be possible to find such a set of formulas.

\section{Semantics}

Which relation $\langle\Theta, M\rangle$ from $A D T_{\imath}$ is denoted by the presentation $\langle\Theta, F\rangle$ in $\operatorname{PRES}_{\imath}(i=\mathbf{2}, \mathbf{3}, 4)$ ? This is a question about the semantics function

$$
\text { sem: } P R E S_{\imath} \rightarrow A D T_{\imath} .
$$

Common semantics are initial and loose from section 2. However, inttial is not appropriate since all models of initial $(S p)$ are isomorphic, and thus, if $m_{1}$ initial $(S p) m_{2}$ and $m_{1}^{\prime}$ initial $(S p) m_{2}^{\prime}$, then $m_{1}$ is isomorphic to $m_{1}^{\prime}$ and $m_{2}$ is isomorphic to $m_{2}^{\prime}$.

Another possible semantics is free. If free $(\langle\Theta, F\rangle)=\langle\Theta, M\rangle$ then structures $m$ in $M$ are required to be free extensions of $\left.m\right|_{\iota_{1}}$ (or $\left.m\right|_{\Theta\left(g_{4}\right)}$ in case 
of $\left.\mathcal{R}_{4}\right)$ with respect to the full subcategory of $\operatorname{Mod}\left(\sum_{i} \Theta\right)(i=\mathbf{2}, \mathbf{3}, 4)$ given by $\{m \mid m \models F\}$ (cf. Goguen and Burstall [10] and Baumeister [2]). free(Sp) can be considered as denoting a partial function from structures of $\operatorname{Mod}(\Theta(1))$ to structures of $\operatorname{Mod}(\Theta(2))$ in the sense that, if for some $m_{1}$ there exists $m_{2}$ such that $m_{1}$ free $(S p) m_{2}$ then $m_{2}$ is unique up to isomorphism. If $\sum_{\imath} \Theta$ does not define any new sorts with respect to $\Theta(1), m_{2}$ is unique.

loose $(S p)$ (or Rel in section 3) in general yields a relation between algebras which can be interpreted as a non-deterministic, partial function on algebras. However, for certain types of $F$ the loose semantics of $S p$ yields a (partial) deterministic function on algebras. This is, for example, the case when $F$ consists of formulas of type $x^{\prime}=t[x]$ for all symbols $x^{\prime}$ not in $\Theta(1)$, where $t[x]$ is a term over the signature $\Theta(1)$, and $\sum_{\imath} \Theta$ does not contain new sorts with respect to $\Theta(1)$. Thus loose semantics allows to specify non deterministic functions as well as deterministic functions.

\section{Related Work}

It is not new or surprising to view states as algebras. For example, Ganzinger [9] and Gurevich [11] use this view to define an algebraic semantics of programming languages. Ganzinger defines the semantics of while-programs to be the composition of a free functor and a forgetful functor on algebras representing the state of the program. In principle we have three specifications. Spec $_{b a s e}$ is the specification of some base types defining the domain over which the variables range. $S p e c_{X}$ is a conservative extension of $S p e c_{\text {base }}$ containing constants of the form $x: s$ denoting the variables of the program. Each program $p$ determines a specification $S p e c_{X \cup X^{\prime}}^{p}$, which is an enrichment of $S_{p e c_{X}}$ that contains for each $x: s$ in Spec $_{X}$ a constant $x^{\prime}: s$ and contains for example in the case where $p$ is the assignment statement $x:=t$ the formula $x^{\prime}=t$. The semantics of a program $p$ is given by $T_{p} ; U_{\sigma}$, where $T_{p}: A \lg \left(\operatorname{Spec}_{X}\right) \rightarrow \operatorname{Alg}\left(\operatorname{Spec}_{X \cup X^{\prime}}^{p}\right)$ is the left adjoint to the forgetful functor $U$ with respect to the inclusion of $S p e c_{X}$ in $S p e c_{X \cup X^{\prime}}^{p}$ and $U_{\sigma}: A \lg \left(\operatorname{Spec}_{X}^{p} \cup X^{\prime}\right) \rightarrow \operatorname{Alg}\left(\operatorname{Spec}_{X}\right)$ is the forgetful functor for the signature morphism $\sigma$ that takes each $x: s$ in $S_{p e c}$ to $x^{\prime}: s$ in $\operatorname{Spec}_{X \cup X^{\prime}}^{p}$. In general $T_{p}$ will be a persistent functor, however, if $p$ does not terminate then $\left(T_{p} ; U\right)(A) \neq A$. This approach corresponds to using the free semantics of section 7 with objects $S p_{p}=\langle\Theta, F\rangle$ from $P R E S_{3}$ such that $\Theta(0)=S p e c_{b a s e}$, $\Theta(1)=\Theta(2)=$ Spec $_{X}$ and $\left\langle\sum_{\mathbf{3}} \Theta, F\right\rangle=$ Spec $_{X}^{p} \cup X^{\prime}$. The only difference is that in our approach, in the case of non-termination, free $\left(S p_{p}\right)$, seen as a function, is undefined; this is the reason why free is defined by using free extensions instead of a free functor. Ganzinger's results can be used in two ways. First, we have new operations at hand to build relations from relations, like the conditional and while construct; on the other hand, the semantics of while-programs have the same semantic domain as their specifications, objects in $A D T_{\mathbf{3}}$. This provides us with a connection of specifications of state transformations to their implementation by imperative programs within the same framework.

In the evolving algebra approach of Gurevich [11] a transformation of an algebra $A$ to an algebra $B$ over the same signature is given by a set of guarded 
local function updates of the form if $b$ then $f(\mathbf{t}):=t_{0}$. The problem in translating these updates in our framework to formulas over $R \operatorname{Sen} 2(\Theta)$ for some object $S p=\langle\Theta, F\rangle$ from $P R E S_{2}$ is the treatment of conflicting updates, eg. $f(a):=$ true and $f(a):=$ false. Gurevich chooses one of the updates non deterministicly and discards the others, while in our approach all updates have to be satisfied. This means that some values have to be equated (eg. true $=$ false) or the relation, viewed as a (non-deterministic) function, is not defined for some algebras. The algebras of Gurevich have only one sort called the superuniverse containing two distinguished elements true and false. Different sorts (universes) are modeled by using characteristic functions on the superuniverse. This allows to model the evolution of sorts just by changing the interpretation of their characteristic functions without having to change the interpretation of the superuniverse.

In the implicit state approach of Dauchy and Gaudel [4] states are enrichments of the datatypes used in the system by access functions. Modifiers are functors on states built from elementary modifiers by sequential and indifferent composition and using conditionals. Elementary modifiers $\mu-a c(\pi, t)$, for some accessor function $a c$ and terms $\pi$ and $t$ over the signature of states, can be translated into our framework as the formula

$$
\forall \mathbf{x}\left(\exists \mathbf{y}:\left(\boldsymbol{\pi}=\mathbf{x} \Rightarrow a c^{\prime}(\mathbf{x})=t\right) \quad \vee \quad \forall \mathbf{y}:\left(\boldsymbol{\pi} \neq \mathbf{x} \Rightarrow a c^{\prime}(\mathbf{x})=a c(\mathbf{x})\right)\right),
$$

where $\mathbf{y}$ is the vector of variables occuring free in $\boldsymbol{\pi}$ and $t$. This is a formula in $F$, where $S p=\langle\Theta, F\rangle$ is an object of $P R E S_{3}, \Theta(1)=\Theta(2)$ contains the accessor function $a c$ and $\Theta(0)$ is the specification of the used datatypes. Now, for a structure $m$ satisfying $S p$, it holds that

$$
a c^{m_{2}}(\mathbf{v})= \begin{cases}\rho^{*}(t) & \text { if } \rho^{*}(\pi)=\mathbf{v} \text { for a variable assignment } \rho: X \rightarrow m_{1} \\ a c^{m_{1}}(\mathbf{v}) \text { else }\end{cases}
$$

This means, that loose $(S p)$ defines a function on algebras corresponding to the semantics of the elementary modifiers of Dauchy and Gaudel. Sequential composition of modifiers is given by the composition of relations in $P R E S_{\mathbf{3}}$ and $A D T_{\mathbf{3}}$ and indifferent composition can be described by colimits in $P R E S_{\mathbf{3}}$ and $A D T_{\mathbf{3}}$, respectively.

In all the above approaches the stress is on constructing transformations of algebras rather than on specifying these transformations in a possibly nonconstructive way. In our approach we are able to specify how the states before and after a transformation are related and not necessarily how this relation is established (eg. by while-programs, local function updates or modifiers).

D-oids are a model theoretic approach by Astesiano and Zucca [1] to define dynamic datatypes. D-oids consist of a set of algebras over some signature together with a set of dynamic operations. A dynamic operation associates to an algebra $A$ and a set of values from $A$ an algebra $B$, a family of functions from the carrier sets of $A$ to the carrier sets of $B$, the tracking map $f: A \Rightarrow B$, and possibly a return value from $B$. However, Astesiano and Zucca do not have a method to specify their dynamic datatypes yet.

All the previous approaches keep the interpretation of sorts fixed, allowing only for the interpretation of function symbols to change. This is not required by 
the d-oids approach. The tracking map $f$ is responsible for relating the elements of the sort whose interpretation has changed in $B$ and also for the elements of those sorts that did not change. In our approach there is the possibility to include new sorts in $\Theta(1)$ and $\Theta(2)$ and functions $f_{i}$ in $\Theta(3), f_{2}: s_{i} \rightarrow s_{i}^{\prime}$, for new sorts $s_{i}$ in $\Theta(1)$ and $\Theta(2)$, and $f_{i}: s_{i} \rightarrow s_{\imath}$, for sorts $s_{i}$ in $\Theta(0)$. The functions $f_{i}$ can be seen as defining the tracking map of dynamic operations.

An approach similar to d-oids is the informal proposal for dynamic abstract datatypes by Ehrig and Orejas [8]. The instant structure specification, a conservative extension of some value type specification, is intended to model class sorts and attribute functions of objects. A dynamic operation denotes for a model $A$ of the instant structure specification, together with a set of values from $A$, a set of transformations from $A$ to some other instant algebra $B$. Given a specification $S p=\langle\Theta, F\rangle$ in $P R E S_{4}$, a value type specification corresponds to $\Theta(0)$, an instant structure specification, roughly, to $\Theta(1)$ and $\Theta(2)$ and certain classes of transformations could be modeled using function symbols $f: s \rightarrow s^{\prime}$ and $f: s \rightarrow s$ in $\Theta(3)$. Then a dynamic operation is an object $R=\left\langle\Theta^{R}, M^{R}\right\rangle$ of $A D T_{4}$ such that $M^{R} \vDash \mathcal{R}^{\mathcal{R}_{4}} F$.

\section{Conclusion}

In this paper we have defined a family of institutions in which abstract datatypes have an interpretation as relations between structures from some base institution. We have achieved two things. First we have a model theory of typed relations, which are objects in $A D T_{2}$, and for typed relations respecting a common environment, objects in $A D T_{\mathbf{3}}$. Since relations are abstract datatypes in certain institutions methods for the construction of abstract datatypes in an arbitrary institution, like eg. various forms of parameterization, can be used to also construct relations, see for example the work of Goguen and Burstall [3, 10], Sannella, Wirsing and Tarlecki [16, 15], Ehrig and others [6]. However, further investigation is needed into whether these constructions are appropriate for constructing relations and which additional constructions, eg. the composition of relations, are needed.

The second achievement of this paper is a specification method for relations between algebras within the theory of abstract datatypes resembling the methods used in specification languages like Z [17], VDM-SL [13] and Larch [12]. Future research has to show how far the resemblance between the presented method and Z, VDM-SL and Larch extends. One could image that the two tiered approach of Larch for some programming language can be given full a semantics within this framework. The base institution is given by the Larch Shared Language; Larch Interface Language specifications are objects in $P R E S_{4}$, which have a denotation as objects in $A D T_{\mathbf{3}}$, and the results of Ganzinger [9] can be used to define the semantics of the programs again as objects of $A D T_{\mathbf{3}}$, which can then be checked to see if they implement the specification.

Colimits in $S I G N$ and therefore in $A D T_{\mathbf{D}}$ and $P R E S_{\mathbf{D}}$ allow us to combine smaller states to larger ones and to extend relations from the smaller states to relations on the larger ones. Also colimits allow us to combine several relations on one state as one relation on the same state. This corresponds to a parallel 
composition of relations instead of a sequential one defined by the composition of relations in section 6.1 .

The interpretation of abstract data types as relations fails when it comes to refinement. The refinement of abstract datatypes does not correspond to the refinement of relations if relations are viewed as non-deterministic functions. The refinement of one abstract datatype by another is an abstract datatype morphism, while $R$ refines to $S$ with respect to a signature morphism $\alpha: \Theta^{R} \rightarrow$ $\Theta^{S}$ iff. $\left.\operatorname{dom}(R) \subseteq \operatorname{dom}(S)\right|_{\alpha}$ and $\alpha:\left.R \rightarrow S\right|_{\alpha(\operatorname{dom}(R))}$ is a morphism between relations. $\left.S\right|_{\alpha(\text { dom }(R))}$ denotes the restriction of $\mathrm{S}$ to the domain $\alpha(\operatorname{dom}(R))$.

\section{References}

1. Egidio Astesiano and Elena Zucca. D-oids: A model for dynamic data-types. Mathematical Structures in Computer Scuence, 1994. to appear.

2. Hubert Baumeister. Unifying initial and loose semantics of parameterized specifications in an arbitrary institution. In Proceedings Tapsoft/CAAP, Brighton, UK, number 493 in LNCS, pages 103-120. Springer, April 1991.

3. R. M. Burstall and J. A. Goguen. The semantics of Clear, a specification language, February 1980.

4. P. Dauchy and M.-C. Gaudel. Algebraic specifications with implicit state, February 1994.

5. R. Diaconescu, J. Goguen, and P. Stefaneas. Logical support for modularisation. Programming Research Group, Oxford University, August 1991.

6. H. Ehrig, P. Pepper, and F. Orejas. On recent trends in algebraic specification. In Proceedings ICALP, Stresa, Italy, number 372 in LNCS, pages 263-288. Springer, July 1989.

7. Hartmut Ehrig and Bernd Mahr. Fundamentals of Algebraic Specification 1: Equations and initial Semantics. Number 6 in EATCS Monographs on Theoretical Computer Science. Springer, 1985.

8. Hartmut Ehrig and Fernando Orejas. Dynamic abstract data types, an informal proposal. Bulletin of the EATCS, (53):162-169, June 1994.

9. Harald Ganzinger. Programs as transformations of algebraic theories (extended abstract). Informatik Fachberichte, 50:22-41, 1981.

10. J. A. Goguen and R. Burstall. Institutions: Abstract model theory for specification and programming. Journal of the Association for Computing Machinery, 39(1):95146, January 1992.

11. Yuri Gurevich. Evolving algebras: An attempt to discover semantics. Bulletin of the EATCS, (43):264-284, February 1991.

12. J. V. Guttag, J. J. Horning, and J. M. Wing. Larch in five easy pieces. Report 5, DEC Systems Research Center, July 1985.

13. Cliff B. Jones. Systematic Software Development Using VDM. International series in computer science. Prentice Hall, New York, 2sd edition, 1990.

14. Saunders Mac Lane. Categorıes for the working mathematıcian. Graduated Texts in Mathematics. Springer, 4th edition, 1988.

15. Donald Sannella and Andrzej Tarlecki. Specifications in an arbitrary institution. Information and Computation, 76(2/3):165-210, February/March 1988.

16. Donald Sannella and Martin Wirsing. A kernel language for algebraic specification. Internal Report CSR-131-83, University of Edinburgh, September 1983.

17. J. Michael Spivey. The $Z$ Notation: A Reference Manual. International series in computer science. Prentice Hall, New York, 2nd edition, 1992. 